\title{
SOLUSI POSITIF DARI SISTEM SINGULAR DISKRIT
}

\author{
BETTY ARYANI \\ Program Studi Matematika, \\ Fakultas Matematika dan Ilmu Pengetahuan Alam, Universitas Andalas, \\ Kampus UNAND Limau Manis Padang, Indonesia, \\ betty.aryani1503@yahoo.com
}

\begin{abstract}
Abstrak. Misalkan matriks $A$ adalah suatu matriks singular. Maka sistem $A \mathbf{x}_{n+1}=$ $B \mathbf{x}_{n}+\mathbf{f}_{n}$ tidak mempunyai solusi. Hal ini disebabkan adanya kondisi awal yang tidak dapat memberikan solusi untuk sistem. Kondisi awal yang dapat memberikan solusi untuk sistem disebut sebagai kondisi awal yang konsisten. Perlu diperhatikan bahwa solusi $\mathbf{x}_{n}$ untuk sistem mungkin positif atau mungkin saja non positif. Solusi $\mathbf{x}_{n}$ dikatakan positif jika $\mathbf{x}_{i} \succ 0$ untuk setiap $i=1,2, \cdots, n$ dan dikatakan non positif jika $x_{i} \preceq 0$ untuk setiap $i=1,2, \cdots, n$. Jika solusi $\mathbf{x}_{n}$ untuk sistem adalah positif maka $\mathbf{x}_{n}$ dikatakan solusi positif dari sistem singular diskrit. Dalam tulisan ini akan diuraikan tentang syarat untuk kepositifan dari solusi sistem singular diskrit dengan menggunakan invers Drazin.

Kata Kunci: Sistem singular diskrit, invers Drazin
\end{abstract}

\section{Pendahuluan}

Diberikan suatu sistem singular diskrit sebagai berikut.

$$
A \mathbf{x}_{n+1}=B \mathbf{x}_{n}+\mathbf{f}_{n}, n \in \mathbb{Z}_{+},
$$

di mana $A, B \in \mathbb{R}^{r \times r}$, dan $\mathbf{x}_{n}, \mathbf{f}_{n} \in \mathbb{R}^{r}$. Notasi $\mathbb{R}^{r \times r}$ menyatakan himpunan matriks-matriks riil berukuran $r \times r, \mathbb{R}_{+}^{r \times r}$ menyatakan himpunan matriks-matriks riil berukuran $r \times r$ yang entri-entrinya non negatif, $\mathbb{R}_{-}^{r \times r}$ menyatakan himpunan matriks-matriks riil berukuran $r \times r$ yang entri-entinya non positif, $\mathbb{R}^{r}$ menyatakan himpunan vektor berdimensi $r, \mathbb{Z}_{+}$menyatakan himpunan bilangan bulat non negatif, dan $\mathbb{C}$ menyatakan himpunan bilangan kompleks. Sistem (1.1) disebut sebagai sistem singular diskrit [5].

Jika $A$ adalah matriks non singular, maka dalam [5] telah diperoleh bahwa solusi dari sistem (1.1) adalah sebagai berikut.

$$
\mathbf{x}_{n}=\left(A^{-1} B\right)^{n} \mathbf{x}_{0}+\sum_{i=0}^{n-1}\left(A^{-1} B\right)^{n-i-1}\left(A^{-1} \mathbf{f}_{i}\right) .
$$

Jika $A$ adalah singular, sistem (1.1) mungkin tidak memiliki solusi. Hal ini disebabkan adanya kondisi awal yang tidak dapat memberikan solusi untuk sistem (1.1). Kondisi awal yang dapat memberikan solusi untuk sistem (1.1) disebut sebagai kondisi awal yang konsisten [5].

Dalam [5], Kaczoreck menyatakan bahwa jika $A$ adalah singular, maka sistem (1.1) mempunyai solusi untuk suatu kondisi awal yang konsisten $\mathbf{x}_{0}$, jika $\operatorname{det}(\lambda A-$ 
$B) \neq 0$ untuk suatu $\lambda \in \mathbb{C}$. Jika kondisi ini terpenuhi, maka solusi sistem (1.1) adalah

$$
\mathbf{x}_{n}=\left(\widehat{A}^{D} \widehat{B}\right)^{n} \widehat{A} \widehat{A}^{D} \mathbf{x}_{0}+\widehat{A}^{D} \sum_{i=0}^{n-1}\left(\widehat{A}^{D} \widehat{B}\right)^{n-i-1} \widehat{\mathbf{f}}_{i}-\left(I-\widehat{A} \widehat{A}^{D}\right) \sum_{i=0}^{k-1}\left(\widehat{A} \widehat{B}^{D}\right)^{i} \widehat{B}^{D} \widehat{\mathbf{f}}_{n+i},
$$

di mana $\widehat{A}=(\lambda A-B)^{-1} A, \widehat{B}=(\lambda A-B)^{-1} B, \widehat{\mathbf{f}_{n}}=(\lambda A-B)^{-1} \mathbf{f}_{n}$ dan $k$ adalah indeks dari matriks $\widehat{A}$.

Perlu diperhatikan bahwa solusi $\mathbf{x}_{n}$ mungkin positif dan mungkin saja non positif. Jika solusi $\mathbf{x}_{n}$ untuk sistem (1.1) adalah positif untuk setiap $n \in \mathbb{N}$, maka sistem (1.1) dikatakan sistem singular diskrit positif. Dalam tulisan ini akan dikaji syarat yang menjamin agar solusi sistem (1.1) adalah positif untuk setiap $n \in \mathbb{N}$.

\section{Solusi Positif dari Sistem Singular Diskrit}

Pada bagian ini akan dikaji tentang bagaimana proses mendapatkan solusi positif dari sistem singular diskrit. Asumsikan bahwa matriks $A$ adalah singular dan $\operatorname{det}(\lambda A-B) \neq 0$ untuk suatu $\lambda \in \mathbb{C}$, maka terdapat $\lambda \in \mathbb{C}$ sedemikian sehingga $(\lambda A-B)^{-1}$ ada.

Dengan mengalikan sistem (1.1) dengan $(\lambda A-B)^{-1}$, diperoleh

$$
\begin{aligned}
(\lambda A-B)^{-1} A \mathbf{x}_{n+1} & =(\lambda A-B)^{-1} B \mathbf{x}_{n}+(\lambda A-B)^{-1} \mathbf{f}_{n} \\
\widehat{A} \mathbf{x}_{n+1} & =\widehat{B} \mathbf{x}_{n}+\widehat{\mathbf{f}}_{n}
\end{aligned}
$$

di mana

$$
\widehat{A}=(\lambda A-B)^{-1} A, \widehat{B}=(\lambda A-B)^{-1} B, \widehat{\mathbf{f}}_{n}=(\lambda A-B)^{-1} \mathbf{f}_{n} .
$$

Lema 2.1. [5] Untuk matriks $\widehat{A}$ dan $\widehat{B}$ yang didefinisikan dalam persamaan (2.2) berlaku,

$$
\widehat{B} \widehat{A}=\widehat{A} \widehat{B}
$$

Bukti. Berdasarkan persamaan (2.2), diperoleh

$$
\begin{aligned}
\lambda \widehat{A}-\widehat{B} & =\lambda(\lambda A-B)^{-1} A-(\lambda A-B)^{-1} B \\
& =(\lambda A-B)^{-1}(\lambda A-B) \\
& =I,
\end{aligned}
$$

atau dapat ditulis

$$
\widehat{B}=\lambda \widehat{A}-I
$$

Akibatnya

$$
\widehat{B} \widehat{A}=(\lambda \widehat{A}-I) \widehat{A}=\widehat{A}(\lambda \widehat{A}-I)=\widehat{A} \widehat{B}
$$

Dalam Lema 2.2 berikut diberikan solusi umum dari sistem singular diskrit.

Lema 2.2. [5] Solusi umum dari persamaan (2.1) adalah

$$
\boldsymbol{x}_{n}=\left(\widehat{A}^{D} \widehat{B}\right)^{n} \widehat{A} \widehat{A}^{D} \boldsymbol{x}_{0}+\widehat{A}^{D} \sum_{i=0}^{n-1}\left(\widehat{A}^{D} \widehat{B}\right)^{n-i-1} \widehat{\boldsymbol{f}}_{i}-\left(I-\widehat{A} \widehat{A}^{D}\right) \sum_{i=0}^{k-1}\left(\widehat{A} \widehat{B}^{D}\right)^{i} \widehat{B}^{D} \widehat{\boldsymbol{f}}_{n+i}
$$


untuk $n \geq 1$, di mana $k=\operatorname{ind}(\widehat{A})$.

Bukti. Untuk membuktikan bahwa (2.3) memenuhi (2.1), akan ditunjukkan bahwa

$$
\widehat{A} \mathbf{x}_{n+1}-\widehat{B} \mathbf{x}_{n}=\widehat{\mathbf{f}}_{n} .
$$

Dengan mengalikan matriks $\widehat{A}$ dengan $\mathbf{x}_{n+1}$, diperoleh

$$
\widehat{A} \mathbf{x}_{n+1}=\left(\widehat{A}^{D} \widehat{B}\right)^{n+1} \widehat{A} \mathbf{x}_{0}+\sum_{i=0}^{n}\left(\widehat{A}^{D} \widehat{B}\right)^{n-i} \widehat{A} \widehat{A}^{D} \widehat{\mathbf{f}}_{i}-\left(I-\widehat{A} \widehat{A}^{D}\right) \sum_{i=0}^{k-1}\left(\widehat{A} \widehat{B}^{D}\right)^{i+1} \widehat{\mathbf{f}}_{n+i+1} .
$$

Dengan mengalikan matriks $\widehat{B}$ dengan $\mathbf{x}_{n}$, diperoleh

$$
\widehat{B} \mathbf{x}_{n}=\left(\widehat{A}^{D} \widehat{B}\right)^{n+1} \widehat{A}_{0}+\sum_{i=0}^{n-1}\left(\widehat{A}^{D} \widehat{B}\right)^{n-i} \widehat{\mathbf{f}}_{i}-\left(I-\widehat{A} \widehat{A}^{D}\right) \sum_{i=0}^{k-1}\left(\widehat{A} \widehat{B}^{D}\right)^{\imath} \widehat{\mathbf{f}}_{n+i}
$$

Selanjutnya,

$$
\begin{aligned}
\widehat{A} \mathbf{x}_{n+1}-\widehat{B} \mathbf{x}_{n}= & \left(\widehat{A}^{D} \widehat{B}\right)^{n+1} \widehat{A} \mathbf{x}_{0}+\sum_{i=0}^{n}\left(\widehat{A}^{D} \widehat{B}\right)^{n-i} \widehat{A} \widehat{A}^{D} \widehat{\mathbf{f}}_{i}-\left(I-\widehat{A} \widehat{A}^{D}\right) \sum_{i=0}^{k-1}\left(\widehat{A} \widehat{B}^{D}\right)^{i+1} \widehat{\mathbf{f}}_{n+i+1} \\
& -\left[\left(\widehat{A}^{D} \widehat{B}\right)^{n+1} \widehat{A}^{D} \mathbf{x}_{0}+\sum_{i=0}^{n-1}\left(\widehat{A}^{D} \widehat{B}\right)^{n-i} \widehat{\mathbf{f}}_{i}-\left(I-\widehat{A} \widehat{A}^{D}\right) \sum_{i=0}^{k-1}\left(\widehat{A} \widehat{B}^{D}\right)^{\imath} \widehat{\mathbf{f}}_{n+i}\right] \\
= & \left(\widehat{A}^{D} \widehat{B}\right)^{n+1} \widehat{A} \mathbf{x}_{0}-\left(\widehat{A}^{D} \widehat{B}\right)^{n+1} \widehat{A}^{D} \mathbf{x}_{0}+\sum_{i=0}^{n}\left(\widehat{A} \widehat{A}^{D} \widehat{B}\right)^{n-i} \widehat{A} \widehat{A}^{D} \widehat{\mathbf{f}}_{i}-\sum_{i=0}^{n-1}\left(\widehat{A}^{D} \widehat{B}\right)^{n-i} \\
& \widehat{\mathbf{f}}_{i}-\left(I-\widehat{A} \widehat{A}^{D}\right) \sum_{i=0}^{k-1}\left(\widehat{A} \widehat{B}^{D}\right)^{i+1} \widehat{\mathbf{f}}_{n+i+1}+\left(I-\widehat{A} \widehat{A}^{D}\right) \sum_{i=0}^{k-1}\left(\widehat{A} \widehat{B}^{D}\right)^{\widehat{\imath}} \widehat{\mathbf{f}}_{n+i} \\
= & \sum_{i=0}^{n}\left(\widehat{A}^{D} \widehat{B}\right)^{n-i} \widehat{A} \widehat{A}^{D} \widehat{\mathbf{f}}_{i}-\sum_{i=0}^{n-1}\left(\widehat{A}^{D} \widehat{B}\right)^{n-i} \widehat{\mathbf{f}}_{i}-\left(I-\widehat{A} \widehat{A}^{D}\right) \sum_{i=0}^{k-1}\left[\left(\widehat{A} \widehat{B}^{D}\right)^{i+1}\right. \\
& \left.\widehat{\mathbf{f}}_{n+i+1}-\left(\widehat{A} \widehat{B}^{D}\right)^{\imath} \widehat{\mathbf{f}}_{n+i}\right] \\
= & \widehat{A} \widehat{A}^{D} \widehat{\mathbf{f}}_{n}+\left(I-\widehat{A} \widehat{A}^{D}\right) \sum_{i=0}^{k-1}\left[\left(\widehat{A} \widehat{B}^{D}\right)^{\imath} \widehat{\mathbf{f}}_{n+i}-\left(\widehat{A} \widehat{B}^{D}\right)^{i+1} \widehat{\mathbf{f}}_{n+i+1}\right] \\
= & \widehat{A} \widehat{A}^{D} \widehat{\mathbf{f}}_{n}+\left(I-\widehat{A} \widehat{A}^{D}\right) \sum_{i=0}^{k-1}\left[\left(\widehat{\mathbf{f}}_{n}-\left(\widehat{A} \widehat{B}^{D}\right) \widehat{\mathbf{f}}_{n+1}\right)+\left(\left(\widehat{A} \widehat{B}^{D}\right) \widehat{\mathbf{f}}_{n+1}-\left(\widehat{A} \widehat{B}^{D}\right)^{2}\right.\right. \\
& \left.\widehat{\mathbf{f}_{n+2}}\right)+\cdots+\left(\left(\widehat{A} \widehat{B}^{D}\right)^{k-2} \widehat{\mathbf{f}}_{n+k-2}-\left(\widehat{A} \widehat{B}^{D}\right)^{k-1} \widehat{\mathbf{f}}_{n+k-1}\right)+\left(\left(\widehat{A} \widehat{B}^{D}\right)^{k-1}\right. \\
& \left.\left.\widehat{\mathbf{f}}_{n+k-1}-\left(\widehat{A} \widehat{B}^{D}\right)^{k} \widehat{\mathbf{f}}_{n+k}\right)\right] \\
= & \left.\widehat{A} \widehat{A}^{D} \widehat{\mathbf{f}}_{n}+\left(I-\widehat{A} \widehat{A}^{D}\right) \widehat{\mathbf{f}}_{n}-\left(\widehat{A} \widehat{B}^{D}\right)^{k} \widehat{\mathbf{f}}_{n+k}\right] \\
= & \widehat{A} \widehat{A}^{D} \widehat{\mathbf{f}}_{n}+\left(I-\widehat{A} \widehat{A}^{D}\right) \widehat{\mathbf{f}}_{n}-\left(I-\widehat{A} \widehat{A}^{D}\right)\left(\widehat{A} \widehat{B}^{D}\right)^{k} \widehat{\mathbf{f}}_{n+k} \\
= & \widehat{A} \widehat{A}^{D} \widehat{\mathbf{f}}_{n}+\widehat{\mathbf{f}}_{n}-\widehat{A} \widehat{A}^{D} \widehat{\mathbf{f}}_{n}-\left(\widehat{A} \widehat{B}^{D}\right)^{k} \widehat{\mathbf{f}}_{n+k}+\left(\widehat{A} \widehat{A}^{D}\right)\left(\widehat{A} \widehat{B}^{D}\right)^{k} \widehat{\mathbf{f}}_{n+k} \\
= & \widehat{A} \widehat{A}^{D} \widehat{\mathbf{f}}_{n}+\widehat{\mathbf{f}}_{n}-\widehat{A} \widehat{A}^{D} \widehat{\mathbf{f}}_{n} \\
= & \widehat{\mathbf{f}}_{n} . \\
&
\end{aligned}
$$

Jadi, solusi (2.3) memenuhi sistem (2.1). 
Dalam Teorema 2.3 berikut diberikan syarat cukup untuk kepositifan dari solusi sistem singular diskrit.

Teorema 2.3. [4] Misalkan $A, B \in \mathbb{R}^{r \times r}$ sedemikian sehingga

(i) Semua elemen diagonal dari matriks $\widehat{A}, \widehat{A}^{D}$ dan $\widehat{B}$ adalah tidak nol,

(ii) $\widehat{A} \preceq 0, \widehat{A}^{D} \preceq 0$, dan $\widehat{B} \preceq 0$,

(iii) $\widehat{\boldsymbol{f}}_{n} \succeq \boldsymbol{O}$ dan $\boldsymbol{x}_{0} \succeq \boldsymbol{O}$.

Misalkan pula

$$
\boldsymbol{x}_{n}=\boldsymbol{L}_{n}\left(\boldsymbol{x}_{0}\right)+\boldsymbol{z}_{k}(n)
$$

di mana

$$
\boldsymbol{L}_{n}\left(\boldsymbol{x}_{0}\right)=\left(\widehat{A}^{D} \widehat{B}\right)^{n} \widehat{A} \widehat{A}^{D} \boldsymbol{x}_{0}+\widehat{A}^{D} \sum_{i=0}^{n-1}\left(\widehat{A}^{D} \widehat{B}\right)^{n-i-1} \widehat{\boldsymbol{f}}_{i},
$$

dan $\boldsymbol{z}_{k}(n)=-\left(I-\widehat{A} \widehat{A}^{D}\right) \sum_{i=0}^{k-1}\left(\widehat{A} \widehat{B}^{D}\right)^{i} \widehat{B}^{D} \widehat{\boldsymbol{f}}_{n+i}$. Maka $\boldsymbol{x}_{n} \succ 0$ untuk $1 \leq n \leq N$, jika vektor $\boldsymbol{x}_{0}$ memenuhi,

$\boldsymbol{x}_{0} \succeq \frac{\left(I-\widehat{A} \widehat{A}^{D}\right) \sum_{i=0}^{k-1}\left(\widehat{A} \widehat{B}^{D}\right)^{i} \widehat{B}^{D} \widehat{\boldsymbol{f}}_{n+i}-\sum_{i=0}^{n-1}\left[\left(\widehat{A}^{D}\right)_{\min }\right]^{n-i}\left[(\widehat{B})_{\min }\right]^{n-i-1} r^{n-i+1} \widehat{\boldsymbol{f}}_{i}}{\left(d_{\max }\left(\widehat{A}^{D}\right)\right)^{n+1}\left(d_{\max }(\widehat{B})\right)^{n} d_{\max }(\widehat{A})}$.

\section{Contoh.}

$$
\begin{gathered}
A=\left(\begin{array}{ccc}
6 & 8 & 9 \\
0 & 0 & 0 \\
12 & 16 & 18
\end{array}\right), B=\left(\begin{array}{ccc}
20 & 2 & 3 \\
-8 & 18 & -10 \\
6 & 6 & 34
\end{array}\right), \mathbf{f}_{n}=\left(\begin{array}{c}
-n-2 \\
-2 n-4 \\
-3-6
\end{array}\right) . \\
\widehat{A}^{D}=\left(\begin{array}{l}
-6.9732 \times 10^{-3}-9.2977 \times 10^{-3}-1.0460 \times 10^{-2} \\
-7.5054 \times 10^{-3}-1.0007 \times 10^{-2}-1.1258 \times 10^{-2} \\
-7.9313 \times 10^{-3}-1.0575 \times 10^{-2}-1.1897 \times 10^{-2}
\end{array}\right) .
\end{gathered}
$$

dan

$$
\widehat{B}^{D}=\left(\begin{array}{c}
-6.794 \times 10^{-3}-9.0295 \times 10^{-3}-1.0158 \times 10^{-2} \\
-7.2889 \times 10^{-3}-9.7466 \times 10^{-3}-1.0933 \times 10^{-2} \\
-7.702 \times 10^{-3}-1.0270 \times 10^{-2}-1.1576 \times 10^{-2}
\end{array}\right)
$$

Untuk $n=1$, diperoleh

$$
\begin{aligned}
\mathbf{x}_{0} & \succeq \frac{\left(I-\widehat{A} \widehat{A}^{D}\right) \widehat{B}^{D} \widehat{\mathbf{f}}_{1}-\left[\left(\widehat{A}^{D}\right)_{\min }\right] r^{2} \widehat{\mathbf{f}}_{0}}{\left(d_{\max }\left(\widehat{A}^{D}\right)\right)^{2}\left(d_{\max }(\widehat{B})\right)\left(d_{\max }(\widehat{A})\right.} \\
& \succeq\left(\begin{array}{c}
1502.2 \\
1687.8 \\
1723.7
\end{array}\right) .
\end{aligned}
$$

Misalkan $\mathbf{x}_{0}=\left(\begin{array}{l}1502.2 \\ 1687.8 \\ 1723.7\end{array}\right)$, sehingga diperoleh

$\mathbf{x}_{1}=\left(\begin{array}{l}1573 \\ 1693 \\ 1789\end{array}\right), \mathbf{x}_{2}=\left(\begin{array}{l}1616 \\ 1739 \\ 1838\end{array}\right), \mathbf{x}_{3}=\left(\begin{array}{l}1659.5 \\ 1786.1 \\ 1887.5\end{array}\right), \mathbf{x}_{4}=\left(\begin{array}{l}1703.4 \\ 1833.4 \\ 1937.5\end{array}\right), \mathbf{x}_{5}=\left(\begin{array}{l}1747.8 \\ 1881.1 \\ 1987.9\end{array}\right)$. 


$$
\begin{gathered}
\text { Misalkan } \mathbf{x}_{0}=\left(\begin{array}{l}
1600 \\
1700 \\
1800
\end{array}\right) \text {, sehingga diperoleh } \\
\mathbf{x}_{1}=\left(\begin{array}{l}
1629.8 \\
1754.1 \\
1853.1
\end{array}\right), \mathbf{x}_{2}=\left(\begin{array}{l}
1674.4 \\
1802.2 \\
1904.5
\end{array}\right), \mathbf{x}_{3}=\left(\begin{array}{l}
1719.6 \\
1850.8 \\
1955.9
\end{array}\right), \mathbf{x}_{4}=\left(\begin{array}{l}
1765.2 \\
1900.0 \\
2007.8
\end{array}\right), \mathbf{x}_{5}=\left(\begin{array}{l}
1811.5 \\
1949.6 \\
2060.3
\end{array}\right) .
\end{gathered}
$$

\section{Ucapan Terima kasih}

Penulis mengucapkan terima kasih kepada Bapak Dr. Muhafzan, Ibu Dr. Ferra Yanuar, Bapak Zulakmal, M.Si, Ibu Izzati Rahmi HG, M.Si yang telah memberikan masukan dan saran sehingga paper ini dapat diselesaikan dengan baik.

\section{Daftar Pustaka}

[1] Anton, H. 1991. Aljabar Linier Elementer Edisi Kedelapan-Jilid 1. Penerbit Erlangga, Jakarta.

[2] Campbell, S.L. 1979. Generalized Inverses of Linear Transformation. Dover. New York

[3] Jacob, B. 1990. Linear Algebra 1. Freeman, W.H. and Company. New York.

[4] Jodar, L dan Merello, P. 2010. Positive Solution of Discrete Dynamic Leontief Input-Output Model with Possibly Singular Capital Matrix. Mathematical and Computer Modelling.

[5] Kaczorek, T. 1992. Linear Control Systems Volume 1. Research Studies Press LTD, England. 